\title{
Capsule summary of NCCN Guidelines for
}

Hodgkin Lymphoma

\author{
Dr. Mary Gamal, MD \\ Lecturer of Internal Medicine, \\ Department of Hematology and Bone Marrow transplantation, \\ Ain Shams University
}

\section{Diagnosis:}

1. Excisional biopsy (recommended) or core biopsy.

2. Immunohistochemistry: Classical HL (CD15 $\left.{ }^{+}, \mathbf{C D}^{+}{ }^{+}, \mathbf{P A X}-5^{+}, \mathrm{CD}^{-}, \mathrm{CD}^{-} 0^{-}, \mathrm{CD}^{-} 5^{-}, \mathrm{CD}^{\circ} \mathrm{a}^{-}\right)$, Nodular lymphocyte-predominant $\left(\mathrm{CD}^{2} \mathrm{O}^{+}, \mathrm{CD}^{+} 5^{+}, \mathrm{CD}^{\circ} \mathrm{a}^{+}, \mathrm{BCL6}^{+}, \mathrm{PAX}-5^{+}, \mathrm{CD} 15^{-}, \mathrm{CD} 30^{-}\right.$, (D3).

\section{Work up:}

1. $\mathrm{CBC}$ with differential, ESR.

2. Comprehensive metabolic panel including LDH and Liver functions.

3. CT, PET-CT, MRI, PET-MRI.

4. Pregnancy test for women in child bearing age.

5. Pulmonary function tests if ABVD or BEACOPP are being used.

6. EF evaluation if doxorubicin-based chemotherapy is indicated.

7. Bone marrow biopsy if there is cytopenias with -ve PET.

8. Pneumococcal, Meningococcal and H-Flu vaccines if splenic radiotherapy is contemplated.

9. HIV, HBV and HCV tests (encouraged).

\section{Staging:}

I. Involvement of a single lymph node region (I) or a localized involvement of a single extra lymphocytic organ or site $\left(\mathrm{I}_{\mathrm{E}}\right)$.

II. Involvement of 2 or more lymph node region on the same side of diaphragm (II) or localized involvement of a single associated extralymphocytic organ or site and its regional lymph node with or without involvement of other regional lymph nodes on the same side of diaphragm $\left(\mathrm{II}_{\mathrm{E}}\right)$.

III. Involvement of lymph node region on both sides of the diaphragm (III) which may be accompanied by localized involvement of an associated extra lymphocytic organ or site (III $)$ or splenic involvement (IIIS) or both $\left(I \mathrm{II}_{\mathrm{E}+\mathrm{S}}\right)$.

IV. Disseminated (multifocal) involvement of one or more extra lymphocytic organ with or without associated lymph node involvement or isolated extra lymphocytic organ involvement with distant (non-regional) lymph node involvement. 
A For no systemic symptoms present.

B For presence of systemic symptoms (B symptoms).

\section{Treatment of Classical HL:}

Stage IA, IIA: Favorable (no bulky disease, $<3$ sites of disease, ESR <50, no E-lesions):

1. ABVD $\times 3$ cycles ------> restage with PET/CT----->

- Deauville 1-2 ------> Follow up or give another ABVD $\times 1$ cycle (total 4).

- Deauville 3-4 ------> ABVD $\times 1$ cycle (total 4) + Involved site irradiation therapy (ISRT) $30 \mathrm{GY}$.

- Deauville 5------> re-biopsy ----->if -ve treat as Deauville 3-4, if +ve treat as refractory disease.

2. ABVD $\times 2$ cycles ------> restage with PET/CT----->

- Deauville 1-2 -----> ABVD $\times 1$ cycle (total 3) + ISRT $30 \mathrm{GY}$

- Deauville 3-4 ------> Escalated BEACOPP $\times 2$ cycles + ISRT $30 \mathrm{GY}$ or ABVD $\times$ 2 cycles (total 4) + ISRT 30 GY.

- Deauville 5------> re-biopsy ----->if -ve treat as Deauville 3-4 , if +ve treat as refractory disease.

3. Stanford V $\times 8$ weeks ------> restage with PET/CT----->

- Deauville 1-4 -----> ISRT $30 \mathrm{GY}$

- Deauville 5------> re-biopsy ----->if -ve ISRT $30 \mathrm{GY}$, if + ve treat as refractory disease.

Stage I, II: Unfavorable ( $>3$ sites of disease, ESR $\geq 50$,+ve B-Symptoms)+ non bulky disease:

1. ABVD $\times 2$ cycles ------> restage with PET/CT----->

- Deauville 1-2 ------> ABVD $\times 2$ cycle (total 4$)+$ ISRT or AVD $\times 4$ cycle (total 6) \pm ISRT.

- Deauville 3-4 ------> Escalated BEACOPP $\times 2$ cycles or ABVD $\times 2$ cycles (total 4) ------> Consired PET/CT ------> ISRT.

- Deauville 5------> re-biopsy ----->if -ve AVD $\times 4$ cycle (total 6) + ISRT, if +ve treat as refractory disease.

2. Stanford V $\times 12$ weeks ------> restage with PET/CT----->

- Deauville 1-4 ------> ISRT to initial sites $>5 \mathrm{~cm}$ (30-36 GY) begins within 2-3 weeks

- Deauville 5------> re-biopsy ----->if -ve ISRT as Deauville 1-4, if +ve treat as refractory disease.

3. Escalated BEACOPP $\times 2$ cycles + ABVD $\times 2$ cycles + ISRT ( for $<60$ years old) $------>$ restage with $\mathrm{PET} / \mathrm{CT}----->$

- Deauville 1-4 ------> ISRT to initial sites $>5 \mathrm{~cm}$ (30-36 GY) begins within 2-3 weeks

- Deauville 5------> re-biopsy ----->if -ve ISRT as Deauville 1-4, if +ve treat as refractory disease. 
Stage I, II: Unfavorable ( $>3$ sites of disease, ESR $\geq 50,+$ ve B-Symptoms)+ bulky mediastinal disease or $>10 \mathrm{~cm}$ adenopathy:

1. ABVD $\times 2$ cycles ------> restage with PET/CT----->

- Deauville 1-3 ------> ABVD $\times 2$ cycle (total 4$)+$ ISRT or AVD $\times 4$ cycle (total 6) \pm ISRT.

- Deauville 4 ------ > Escalated BEACOPP $\times 2$ cycles + ISRT or ABVD $\times 2$ cycles (total 4) + ISRT.

- Deauville 5------> re-biopsy ----->if -ve treat as Deauville 4, if +ve treat as refractory disease.

2. Stanford V $\times 12$ weeks ------ > treat as Stage I, II: Unfavorable non bulky.

3. Escalated BEACOPP $\times 2$ cycles + ABVD $\times 2$ cycles + ISRT (for $<60$ years old) ----->treat as Stage I, II: Unfavorable non bulky.

Stage III, IV:

1. ABVD $\times 2$ cycles ------> restage with PET/CT----->

- Deauville 1-3 ------> ABVD $\times 4$ cycles ------> Follow up or ISRT to initially bulky or PET + ve sites.

- Deauville 4-5 ------>

○ Escalated BEACOPP $\times 4$ cycles or ------> restage with PET/CT----->

- Deauville 1-3 ------> Follow up or ISRT to initially bulky or PET + ve sites.

- Deauville 4-5 ------>re-biopsy ----->if -ve ISRT to initially bulky or PET + ve sites, if +ve treat as refractory disease.

-

○ ABVD $\times 2$ cycles (total 4)------> restage with PET/CT----->

- Deauville 1-3 ----->ABVD $\times 2$ cycle (total 6) \pm ISRT to initially bulky or PET +ve sites.

- Deauville 4-5 ------>re-biopsy ----->if-ve ABVD $\times 2$ cycle (total 6) \pm ISRT to initially bulky or PET + ve sites, if +ve treat as refractory disease.

2. Stanford V $\times 12$ weeks ( in selected patients with IPS $<3$ ) ------ > restage with $\mathrm{PET} / \mathrm{CT}$----->

- Deauville 1-4 ------> ISRT to initial sites $>5 \mathrm{~cm}$, involved spleen (30-36 GY) begins within 2-3 weeks

- Deauville 5------> re-biopsy ----->if -ve ISRT as Deauville 1-4, if +ve treat as refractory disease.

3. Escalated BEACOPP $\times 6$ cycles + ABVD $\times 2$ cycles + ISRT ( for $<60$ years old) -----> re-stage with $\mathrm{PET} / \mathrm{CT}----->$

- Deauville 1-2 ------> Follow up

- Deauville 3-4------> ISRT to residual PET +ve sites $>2.5 \mathrm{~cm}$ and Follow up.

- Deauville 5 ------> re-biopsy ----->if -ve Follow up or ISRT to initially bulky or $\mathrm{PET}+\mathrm{ve}$ sites, if + ve treat as refractory disease.

\section{Treatment of Classical HL Refractory disease:}

Biopsy proven refractory disease ------> Second line chemotherapy (ABVD, CHOP, CVP) + Rituximab ------> re-stage with PET/CT---> 
- Deauville 1-3 ------> High-dose therapy + autologous stem cell rescue (HDT/ASCR $\pm R T$ ) or Follow up \pm RT (if HDT/ASCR contraindicated).

- Deauville 4-----> HDT/ASCR \pm RT or RT or Additional chemotherapy \pm RT

- Deauville 5 ------> RT or Additional chemotherapy \pm RT

\section{Treatment of Nodular Lymphocyte-Predominant HL:}

$>$ Initial treatment according to the stage:

CS IA, IIA (non-bulky): Follow up or ISRT.

CS IB, IIB or CS IA, IIA (bulky): chemotherapy (ABVD, CHOP, CVP) + Rituximab+ ISRT.

CS IIIA, IVA: chemotherapy (ABVD, CHOP, CVP) + Rituximab \pm ISRT or Rituximab or Local RT (Palliation of locally symptomatic disease).

CS IIIB, IVB: chemotherapy (ABVD, CHOP, CVP) + Rituximab \pm ISRT.

$>$ For all stages ----->Re-evaluation with PET-CT:

- Response: Follow up or ISRT (if no prior RT).

○ Stable or progressive: Rebiopsy-----> >if -ve Follow up, if +ve treat as refractory disease.

\section{Treatment of Nodular Lymphocyte-Predominant Refractoty HL:}

Biopsy proven refractory disease ------>

- Aggressive B-cell lymphoma: treat as B-cell lymphoma.

- NLPHL: Follow up or Rituximab \pm chemotherapy \pm ISRT ----->Re-evaluation with PETCT----->If clinical response follow up, if progressive disease treat as refractory classical HL.



